\author{
Marquette University \\ e-Publications@Marquette
}

$10-2007$

\title{
Rapid Detection of Analytes with Improved Selectivity Using Coated Microcantilever Chemical Sensors and Estimation Theory
}

\author{
Michael J. Wenzel \\ Marquette University \\ Fabien Josse \\ Marquette University, fabien.josse@marquette.edu \\ Edwin E. Yaz \\ Marquette University, edwin.yaz@marquette.edu \\ Stephen M. Heinrich \\ Marquette University, stephen.heinrich@marquette.edu \\ P. G. Datskos \\ Oak Ridge National Laboratory
}

Follow this and additional works at: https://epublications.marquette.edu/civengin_fac

Part of the Civil and Environmental Engineering Commons

\section{Recommended Citation \\ Wenzel, Michael J.; Josse, Fabien; Yaz, Edwin E.; Heinrich, Stephen M.; and Datskos, P. G., "Rapid Detection of Analytes with Improved Selectivity Using Coated Microcantilever Chemical Sensors and Estimation Theory" (2007). Civil and Environmental Engineering Faculty Research and Publications. 58. https://epublications.marquette.edu/civengin_fac/58}


Marquette University

e-Publications@Marquette

\section{Civil and Environmental Engineering Faculty Research and Publications/College of Engineering}

This paper is NOT THE PUBLISHED VERSION; but the author's final, peer-reviewed manuscript. The published version may be accessed by following the link in the citation below.

SENSORS, 2007 IEEE, (October 28-31, 2007): 91-94. DOI. This article is (C) The Institute of Electrical and Electronics Engineers and permission has been granted for this version to appear in $\underline{\mathrm{e}-}$ Publications@Marquette. The Institute of Electrical and Electronics Engineers does not grant permission for this article to be further copied/distributed or hosted elsewhere without the express permission from The Institute of Electrical and Electronics Engineers.

\section{Rapid Detection of Analytes with Improved Selectivity Using Coated Microcantilever Chemical Sensors and Estimation Theory}

M. J. Wenzel

Marquette University, Milwaukee, WI

F. Josse

Marquette University, Milwaukee, WI

E. Yaz

Marquette University, Milwaukee, WI

S. M. Heinrich

Marquette University, Milwaukee, WI

P. G. Datskos

Oak Ridge National Laboratory, Oak Ridge, TN 


\section{Abstract:}

Rapid detection of analytes with improved selectivity is achieved though the use of estimation theory to analyze the response of polymer-coated microcantilever chemical sensors. In general, chemical sensors exhibit partial selectivity and can have relatively long response times. Using estimation theory, it is possible to make shortterm response predictions from past data. This makes it possible to use the transient information (response time), often unique to an analyte/coating pair, to achieve an improvement in analyte species recognition while simultaneously allowing for a reduction in the time required for identification and quantification. An extended Kalman filter is used as a recursive online approach to refine the estimate of the sensor's future response. Both identification and quantification are thus possible as soon as the filter estimate achieves a high confidence level. Also, with improved selectivity, identification is possible using fewer sensors in an array.

\section{SECTION I. Introduction}

In general, chemical sensors exhibit partial selectivity, with a single sensor responding to many different chemical analytes. However, when exposed to an array of many chemical sensors, each functionalized by a different coating, a specific analyte will have its own unique response (a chemical fingerprint) [1], [2]. The sensor array technique makes it possible to distinguish a target analyte from interferents, thus greatly reducing the false alarm rate. However, developing coatings which maximize the array's ability to detect the target analyte is very time consuming and costly. Furthermore, relatively long response times of several minutes have been observed, especially in direct liquid-phase detection. Thus, there is a need to improve array selectivity while simultaneously allowing for a reduction in the time required for identification and quantification.

Typically, in analyzing sensor arrays, only the equilibrium response of each sensor is used to identify the analyte. However, it has been shown that use of the sensor's transient behavior can significantly improve classification results [3]. Indeed, the transient behavior, which is related to the sorption processes, can be unique to an analyte/coating pair.

In this work, the extended Kalman filter is applied to analyze state-space models of the bending response of polymer-coated microcantilever chemical sensors, taking into account relaxation and plasticization effects of the coating. The result is to achieve online estimation of the complete sensor response, including the transient and equilibrium responses, well before the steady-state is observed. The transient response information is then used to improve selectivity of the sensor array over using only the equilibrium response of the sensor.

\section{SECTION II. Theory}

\section{A. State-Space Modeling}

In order to extract the transient information, accurate models of the sensor response are necessary. These models must include well-known effects of polymer coatings, such as sorption-induced elongation, coating plasticization, and polymer relaxation.

When analyte interacts with the coating on the microcantilever, the analyte may undergo surface adsorption or bulk absorption. Absorption is common for relatively thick polymer coatings, whereas adsorption is generally more common in sensors coated with thin metals or bio-receptors. In the case of adsorption electrostatic and steric effects cause a surface stress to develop on the functionalized side of the coating resulting in cantilever bending. In this case, a form of Stoney's equation can be used to relate the total deflection, $Z_{L}$, to the surface stress generated by adsorption [4],

$Z_{L}=\frac{3 L^{2}\left(1-v_{1}\right)}{E_{1} h_{1}^{2}} \sigma_{S},(1)$ 
where $E_{1}, v_{1}$, and $h_{1}$ are the modulus, Poisson's ratio, and thickness of the substrate, respectively, and $L$ is the length of the microcantilever. The surface stress, $\sigma_{S}$, is interpreted as a traction on the microcantilever having units of force per length $(\mathrm{N} / \mathrm{m})$. In the case of bulk absorption, expansion of the coating, which is attached to the substrate, causes stress to build up in the coating and thus bending of the cantilever. The deflection can be expressed in terms of sorption-induced elongation, $\Delta \varepsilon$, (i.e. the expansion sorption would cause if the coating was not attached to the substrate) by the bending equation [5],

$Z_{L}=3 L^{2}\left(\frac{h_{1}+h_{2}}{h_{2}^{2} K}\right) \Delta \varepsilon,(2)$

with

$K=4+6\left(\frac{h_{1}}{h_{2}}\right)+4\left(\frac{h_{1}}{h_{2}}\right)^{2}+\left(\frac{E_{1}}{E_{2}}\right)\left(\frac{h_{1}}{h_{2}}\right)^{3}+\left(\frac{E_{2}}{E_{1}}\right)\left(\frac{h_{2}}{h_{1}}\right)$,

where $E_{2}$ and $h_{2}$ are the modulus and thickness of the coating, respectively. Note that the bending equation, (2), relies on the assumption that the coating is an elastic material with a modulus that does not change significantly during detection.

In order to develop a state-space model the bending equation is used as the output equation combined with a dynamic model for the absorption process. For example, if sorption is assumed first-order then the governing differential equation is,

$C(t)=\left(1 / \tau_{s}\right)\left(K_{p} C_{a m b}(t)-C(t)\right),(3)$

where $C(t)$ is the sorbed analyte concentration, $C_{a m b}(t)$ is the ambient analyte concentration, $K_{p}$ is the partition coefficient, and $\tau_{s}$ is the sorption time constant. Here, the dot notation is used to represent the time derivative. Equations (2) and (3) are combined in the form of a state-space model by assuming that, for the small concentration ranges during detection, the sorption-induced elongation is proportional to the sorbed analyte concentration. In that case, the state variable for the system is the sorbed analyte concentration, $C(t)$.

The previous model can be used to extract the transient information from sensors that exhibit a first-order response. However, atypical responses that do not exhibit first-order behavior have been observed in microcantilever sensor data [6]. In order to describe these responses more sophisticated models are necessary. These models must include the effects of plasticization and stress relaxation.

Plasticization of the polymer coating upon analyte sorption is known to cause changes in the modulus of the coating. In most cases this effect softens the polymer, reducing the modulus; however, in rare cases the opposite is possible. To incorporate the effects of coating plasticization the previous model can be used by replacing the modulus with the concentration dependent modulus $E_{2}(C(t))$. This causes the state-space equations to be nonlinear. For a small range of concentrations, the modulus can be assumed a linear function of the concentration, such that

$E_{2}(C(t))=E_{2,0}+\delta C(t),(4)$

where $E_{2,0}$ is the modulus before analyte sorption and $\delta$ is the change in modulus per change in sorbed analyte concentration. Note that for the common case of coating softening $\delta$ is negative. 
Stress relaxation is a common effect of a polymer coating due to the viscoelastic properties of polymers. In a viscoelastic material the stress is dependent on the strain history rather than just the strain at that point in time. This can be described by the three-parameter solid model [7], [8],

$\varepsilon(t)+\tau_{r} \varepsilon(t)=J_{R} \sigma(t)+\tau_{r} J_{U} \sigma(t),(5)$

where $J_{U}$ is the unrelaxed compliance, $J_{R}$ is the relaxed compliance, and $\tau_{r}$ is the polymer time constant given a constant stress. For a constant stress the strain in a viscoelastic material will increase with time (i.e. creep) and for a constant strain the stress will decrease with time (i.e. relaxation). This behavior is shown in Fig. 1. When the device is coated with a polymer, the substrate is generally much stiffer than the coating, i.e. $E_{1} \gg 1 / J_{U}$ In this case, the relaxation effect can be incorporated into a second-order state-space model using the sorbed analyte concentration and the stress in the coating, $\sigma_{2}$, as the state variables and the deflection at the tip of the cantilever as the output. This is given by $(3)$ and

$$
\begin{gathered}
\sigma_{2}(t)=\left(\begin{array}{c}
\lambda\left(\tau_{s}-\tau_{r}\right) / \tau_{s} \tau_{r} J_{U} \\
+
\end{array}\right)(t)-\left(J_{R} / J_{U} \tau_{r}\right) \sigma_{2}(t) \\
+\left(K_{p} \lambda / \tau_{s} J_{U}\right) C_{a m b}(t), \\
Z_{L}(t)=\left(\frac{3 L^{2} h_{2}\left(h_{1}+h_{2}\right)}{E_{1} h_{1}^{3}}\right) \sigma_{2}(t)
\end{gathered}
$$

where $\lambda$ is the constant of proportionality between the sorbed analyte concentration and sorption-induced elongation.

\section{B. Analysis and Signal Processing}

The Extended Kalman Filter (EKF) is a state variable estimation technique that is capable of online, real-time state estimation for nonlinear systems [9]. The EKF can be used for parameter estimation by first converting the state-space models to discrete time using any of the possible methods; the simplest of which is the Euler approximation that approximates a values time derivative by the difference between two time adjacent samples divided by the sampling time. Then the parameters must be augmented with the state-vector [10]. Note that this causes a linear system to become nonlinear. For this reason the EKF must be used and not the original Kalman filter.

Standard curve fitting techniques require the data to be presented in batch format. The advantages that the EKF has over these other techniques are that the EKF can handle sequential data without the necessity of storing old data in memory, thus allowing data analysis to be done on simple microprocessors rather than computers. Moreover, the EKF can account for noise in the system, if necessary, and has the potential to be extended to binary and tertiary mixtures of analytes.

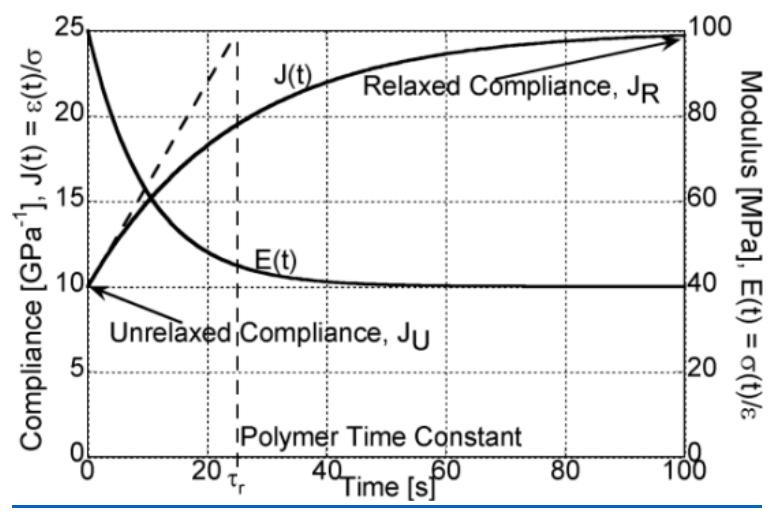


Figure 1. The compliance (and strain) for a constant stress and the modulus (and stress) for a constant strain in an example viscoelastic material with $J_{U}=10 \mathrm{GPa}^{-1}, J_{R}=25 \mathrm{GPa}^{-1}$, and $\tau_{r}=25 \mathrm{~s}$.

An initial estimate of the parameters along with the uncertainty of these estimates is required for the extended Kalman filter. After the initial conditions are specified the EKF will update the parameters and their respective uncertainties with each new sample obtained. However, if the initial estimates of the parameters are not sufficiently close to their actual values, the EKF may converge slowly or even diverge [9]. In order to quickly obtain parameter estimates so that detection can be assessed early, good initial conditions are required. There are three ways that this can be handled. If there is sufficient a priori knowledge of the system then this knowledge can be used to determine parameter estimates. Otherwise, it may be desired to run multiple filters each with different initial conditions or to use a curve fitting technique on the first few samples in order to obtain these initial conditions.

The model parameters contain the transient and steady-state information of the sensor response. Once the uncertainty in the parameters is sufficiently low they can be used in order to perform analyte recognition. This is generally possible well before the equilibrium response of the sensor has been reached. Furthermore, the transient information can also be unique to an analyte/coating pair. The transient information, in addition to the steady-state response, as estimated before reaching equilibrium, can then be used during pattern recognition and feature extraction.

\section{SECTION III. Experimental Procedure}

The extended Kalman filter technique was applied to a data set provided by Oak Ridge National Laboratory and published in Ref [6]. The data consisted of microcantilevers each coated with a different monomer/polymer layer on a functionalized gold layer. All sensors were placed in the same flow cell. Thus, all sensors were exposed to the same conditions. The coated microcantilevers were simultaneously exposed to nine different analytes. This was repeated 4 or 8 times for each analyte. The microcantilever coatings and the analytes are shown in Table 1. For more information concerning the coating procedures and experimental setup see Ref [6].

The EKF was applied to each of the responses in the data set. In order to demonstrate the selectivity of the array using the transient information, regularized linear discriminants analysis (RLDA) was used [11]. This allows the data to be projected onto a two dimensional space while still retaining most of the identification potential. RLDA was used because of the high dimensionality of the data and small training set. The RLDA algorithm was trained using four samples from each analyte. The others were saved for verification. Note that the verification set and the training set were collected on different days.

\section{SECTION IV. Results and Discussion}

For responses exhibiting typical first-order behavior the EKF was capable of obtaining good estimates for both the transient and steady-state parameters in about 8-10 seconds; much faster then the time required to reach equilibrium. Slightly longer time was usually necessary for responses exhibiting relaxation or plasticization. Fig. 2 shows the prediction of responses (one first-order and one exhibiting relaxation) using the parameters estimated at 10 and 20 seconds. As more data is obtained the estimates become more accurate.

Also depicted on Fig. 2 is the sorption time defined as the time required for the coating to absorb $63 \%$ of the equilibrium concentration. Fig. 2 shows the response of a CD-coated microcantilever sensor to two different analytes. The sorption times (as estimated by the EKF) have significantly different values for the two analytes. These sorption times can be as repeatable as the steady-state value. Thus, the sorption time is in many cases unique to an analyte/coating pair. 
The extracted sorption times, in addition to the equilibrium responses, can be used to perform analyte recognition. Utilizing this additional information, the number of sensors in the array can be reduced with little loss of the ability to perform recognition. The RLDA plots are shown in Fig. 3 (both training and test data are plotted). The analytes remain well clustered, and identification is still possible, when using only 3 sensors. This is clearly not the case if only the equilibrium response is used. It is also noted, but not shown here that when using only the equilibrium response, the clusters seem to drift from day to day making retraining necessary. This is not a problem when using the transient response.
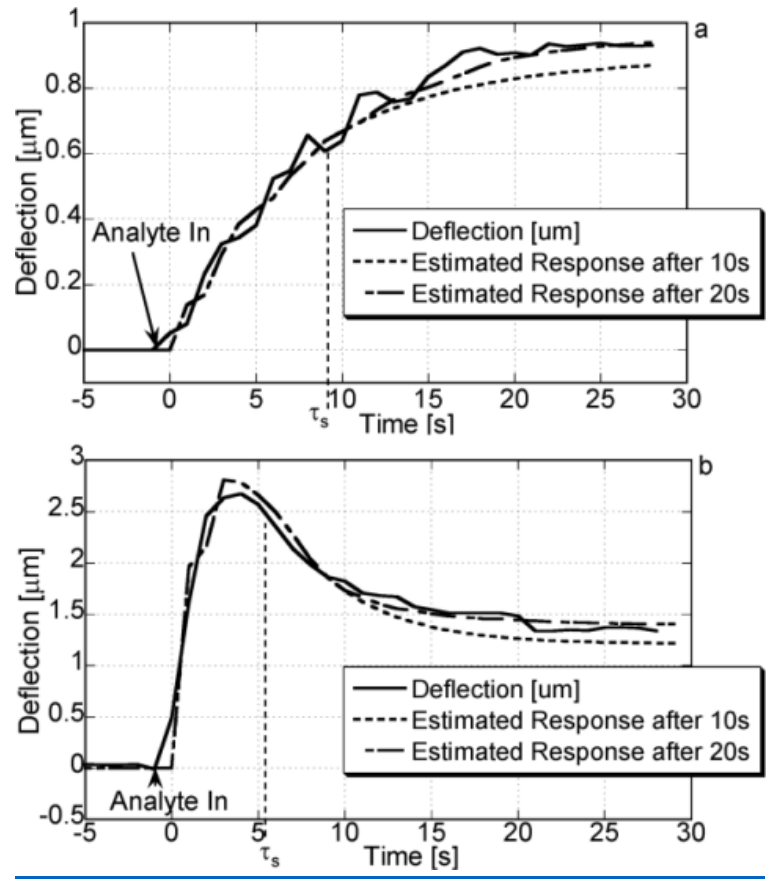

Figure 2. Estimated output obtained using an extended Kalman filter. Predictions were done using the first 10 (20) seconds of measured data. a) detection of 2-propanol, b) detection of tricloroethylene using a CD-coated microcantilever sensor. Sorption times are also depicted.

TABLE I. COATINGS AND ANALYTES USED

\begin{tabular}{|l|l|}
\hline Coating & Abbrev. \\
\hline 4-tert-Butylcalix [6]arene & Cal-6 \\
\hline Poly(diphenoxyphosphazene) & PDPP \\
\hline Heptakis(6-0-tert-butyl dimethylsily1-2,3 di-O-acetyl)- $\beta$-CD & CD \\
\hline Propanethiol treated gold & BL1 \\
\hline Squalane & Squ \\
\hline Copper Phthalocyanine & CuPc \\
\hline Propanethiol treated gold & BL2 \\
\hline Analyte & Abbrev. \\
\hline 1-Butanol & BUT \\
\hline Chloroform & CHLO \\
\hline Dichloromethane & DCL \\
\hline Diisopropylmethylphosphonate & DIMP \\
\hline Ethanol & ETH \\
\hline Methanol & METH \\
\hline 2-Propanol & IPA \\
\hline
\end{tabular}




\begin{tabular}{|l|l|}
\hline Trichloroethylene & TCE \\
\hline Tetrachloroethylene & TRCE \\
\hline
\end{tabular}

\section{SECTION V. Conclusion}

This work has demonstrated a technique for improving both the selectivity and analyte identification time for arrays of coated microcantilever chemical sensors. The extended Kalman filter was used in order to extract transient and steady-state sensor response information well before equilibrium is reached.

The presented technique can be extended to any sensor platform, provided that the model is changed to fit the transduction principles. A greater improvement in identification time is expected in the liquid-phase, where sensors can take several minutes to reach equilibrium.
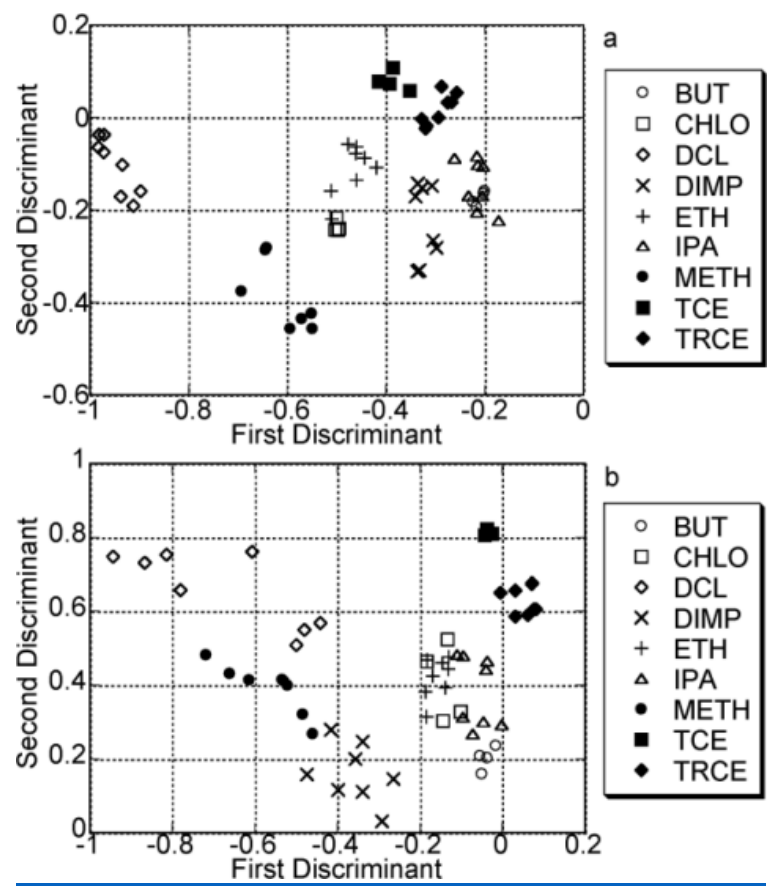

Figure 3. Scatter plots including transient information from the sensor response. a) using the seven polymer coated microcantilevers b) using only three polymer coated microcantilevers.

\section{ACKNOWLEDGMENT}

Special thanks to M. J. Sepaniak for providing the experimental data and helpful discussion.

\section{REFERENCES}

1.K. Albert, N. Leqis, C. Schauer, G. Sotzing, S. Stitzel, T. Vaid, et al., "Cross-reactive chemical sensor arrays", Chem. Rev., vol. 100, pp. 2595-2626, 2000.

2.J. W. Gardner and P. N. Bartlett, Electronic Noses: Principles and Applications, Oxford University Press, 1999.

3.C. Di Nucci, A. Fort, S. Rocchi, L. Tondi, V. Vignoli, F. Di Francensco, et al., "A measurement system for odor classification based on the dynamic response of QCM sensors", IEEE T. Instrum. Meas., vol. 52, no. 4, 2003.

4.N. V. Lavrik, M. J. Sepaniak and P. G. Datskos, "Cantilever transucders as a platform for chemcial and biological sensors", Rev. Sci. Instrum, vol. 75, no. 7, July 2004.

5.Z. Hu, T. Thundat and R. J. Warmack, "Investigation of adsorption and absorption-induced stresses using microcantilever sensors", J. Appl. Phys., vol. 90, no. 1, pp. 427-431. 
6.L. R. Senesac, P. Dutta, P. G. Datskos and M. J. Sepaniak, "Analyte species and concentration identification using differentially functionalized microcantilever arrays and artificial neural networks", Anal. Chim. Acta, vol. 558, pp. 94-101, 2006.

7.N. G. McCrum, B. E. Read and G. Williams, Anelastic and Dielectric Effects in Polymeric Solids, John Wiley, 1967.

8.W. Flügge, Viscoelasticity, Springer-Verlag, 1975.

9.K. Reif, S. Günther, E. Yaz and R. Unbehauen, "Stochastic stability of the discrete-time extended Kalman filter", IEEE T. Automat. Contr., vol. 44, no. 4, 1999.

10.L. Ljung, "Asymptotic Behavior of the extended Kalman filter as a parameter estimator for linear systems", IEEE T. Automat. Contr., vol. AC-24, no. 1, pp. 36-50, 1979.

11.J. H. Friedman, "Regularized discriminant analysis", J. Am. Stat. Assoc., vol. 84, pp. 165-175, 1989. 